\title{
NEW MODELING OF STEADY-STATE MODES OF COMPLEX ELECTRICAL GRIDS OF POWER SYSTEMS
}

\author{
Arman Akhmetbayev ${ }^{1}$,Dauren Akhmetbayev ${ }^{\mathrm{a} 2}$, Serik Zhumazhanov², \\ Bauyrzhan Zhakishev ${ }^{2}$ \\ ${ }^{1}$ Directorate of Information Systems of "Kaztelecom" JSC, Almaty, Kazakhstan \\ ${ }^{2}$ Department of "Electrical Power Supply" KATU n.a. S. Seifulullin, Astana, Kazakhstan
}

\begin{abstract}
Classical methods for modeling the steady-state modes of complex electrical networks and systems are based on the application of nonlinear node equations. Nonlinear equations are solved by iterative methods, which are connected by known difficulties. To a certain extent, these difficulties can be weakened by applying topological methods. In this paper, we outline the theoretical foundations for the formation of the inverse form of nodal stress equations based on the topology of electrical networks and systems. A new topological method for calculating the distribution coefficients of node currents is proposed based on all possible trees of a directed graph of a complex electrical network. A complex program for calculating current distribution coefficients and forming steady-state parameters in the MATLAB environment has been developed.
\end{abstract}

\section{Introduction}

When analyzing the functioning and management of power system regimes, it becomes necessary to perform a large amount of work on the calculation of steady-state regimes [1]. Equations of steady-state regimes of complex energy systems are nonlinear and can be solved only by iterative methods. The difficulties of obtaining real solutions of non-linear equations of steady-state regimes can be overcome if one approaches from the point of application of the inverse form of nodal equations [2]. There exist several indirect methods for determining the matrix of nodal resistances, which in the general case did not lead to the desired result with respect to complex systems. In [3], topological methods were developed for calculating electric power systems in which the steady-state regime was directly formalized from the topology of the circuit, which turned out to be of little use for a complex system. Below we propose an improved topological method for modeling the steady-state regimes of complex electric power systems.

aCorresponding author: Axmetbaev46@mail.ru 


\section{The transformation of the node equation}

The inverse form of the node equations is as follows:

$$
\dot{U}_{\Delta}=\underline{Z \dot{J}},
$$

where $\underline{Z}=\underline{Y}^{-1}$-node impedance matrix; $\underline{Y}^{-1}$-square matrix of nodal conductivities;

$$
\underline{J}=-\frac{\hat{S}}{\sqrt{3} \underline{\hat{U}}}-\text { column matrix of given current. }
$$

The analytical relationship between the given currents and the distribution of currents in the passive circuit is determined by the expressions:

$$
\begin{aligned}
& M \underline{\dot{I}}=\underline{\dot{J}} \\
& \dot{I}=\underline{C} \dot{j},
\end{aligned}
$$

where $\underline{C}$-rectangular matrix of current distribution coefficients; $M$-the first incidence matrix.

Expression (1) with provision for (2), (3) can be written down as follows:

$$
\underline{\dot{U}}_{\Delta}=\underline{C}^{t} \underline{Z}_{b} \underline{C} \dot{J},
$$

where $\underline{C}^{t}=\underline{Z} M \underline{Z}_{b}^{-1}$-transposed matrix of current distribution coefficients.

The obtained equation (5) allows us to write the identity as follows:

$$
\underline{Z}=\underline{C}^{t} \underline{Z}_{b} \underline{C}=\underline{Y}^{-1}
$$

Thus, the problem of calculating the matrix of nodal resistances is reduced to determining the matrix of the distribution coefficients of the given currents, which substantially reduces the amount of computing work.

\section{Current distribution coefficients and electrical network topology}

The topology of electrical networks makes it possible to establish an analytical relationship between the current distribution coefficients and the structure of the linear graph of the network. A significant place in the network topology is occupied by such subgraphs as trees and 2-trees. From the values of trees and 2-trees of the graph numerators and denominators of the distribution coefficients of the driving currents of electric networks are formed [3].

In the works $[4,5]$, a new approach to the determination of the numerators of the current distribution coefficients in the function of the network parameters was proposed without searching for and determining the value of the 2-trees of the graph of a complex network.For the closed network shown in Figure 1a, the first column of the matrix of the distribution coefficients of the node currents is determined by solution of the equation

$$
\underline{I}_{k}=\underline{Z}_{1}+\underline{Z}_{2}+\underline{Z}_{3}+\underline{Z}_{4}=\underline{Z}_{1}
$$

generated for the circuit (see Figure $1 b$ ). 


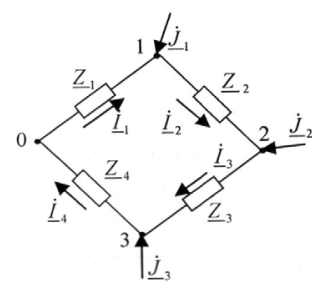

a

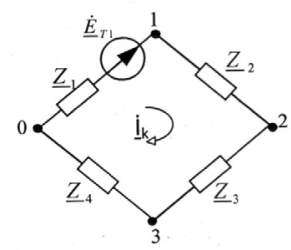

$\mathrm{b}$

Fig. 1.The closed circuit layout: a) original circuit; b) design circuit.

$$
\underline{C}=\frac{1}{\underline{Z}_{k}}\left|\begin{array}{ccc}
-\left(\underline{Z}_{2}+\underline{Z}_{3}+\underline{Z}_{4}\right) & -\left(\underline{Z}_{3}+\underline{Z}_{4}\right) & -\underline{Z}_{4} \\
\underline{Z}_{1} & -\left(\underline{Z}_{3}+\underline{Z}_{4}\right) & -\underline{Z}_{4} \\
\underline{Z}_{1} & \left(\underline{Z}_{1}+\underline{Z}_{2}\right) & -\underline{Z}_{4} \\
\underline{Z}_{1} & \left(\underline{Z}_{1}+\underline{Z}_{2}\right) & \left(\underline{Z}_{1}+\underline{Z}_{2}+\underline{Z}_{3}\right)
\end{array}\right|,
$$

where $\underline{Z}_{k}=\underline{Z}_{1}+\underline{Z}_{2}+\underline{Z}_{3}+\underline{Z}_{4}-$ loop resistance.

The topological properties of the current distribution coefficients (7) can be determined by formulas that can be written down after simple transformations as follows:

$$
\begin{aligned}
& \underline{C}=\frac{1}{\underline{Y}_{1} \underline{Y}_{2} \underline{Y}_{3}+\underline{Y}_{1} \underline{Y}_{2} \underline{Y}_{4}+\underline{Y}_{1} \underline{Y}_{3} \underline{Y}_{4}+\underline{Y}_{2} \underline{Y}_{3} \underline{Y}_{4}} \times \\
& \left|\begin{array}{ccc}
-\underline{Y}_{1}\left(\underline{Y}_{2} \underline{Y}_{3}+\underline{Y}_{2} \underline{Y}_{4}+\underline{Y}_{3} \underline{Y}_{4}\right) & -\underline{Y}_{1}\left(\underline{Y}_{2} \underline{Y}_{3}+\underline{Y}_{2} \underline{Y}_{4}\right) & -\underline{Y}_{1}\left(\underline{Y}_{2} \underline{Y}_{3}\right) \\
\underline{Y}_{2}\left(\underline{Y}_{3} \underline{Y}_{4}\right) & -\underline{Y}_{2}\left(\underline{Y}_{Y_{3}}+\underline{Y}_{1} \underline{Y}_{4}\right) & -\underline{Y}_{2}\left(\underline{Y}_{1} \underline{Y}_{3}\right) \\
\underline{Y}_{3}\left(\underline{Y}_{2} \underline{Y}_{4}\right) & \underline{Y}_{3}\left(\underline{Y}_{Y_{4}}+\underline{Y}_{2} \underline{Y}_{4}\right) & -\underline{Y}_{3}\left(\underline{Y}_{1} \underline{Y}_{2}\right) \\
\underline{Y}_{4}\left(\underline{Y}_{2} \underline{Y}_{3}\right) & \underline{Y}_{4}\left(\underline{Y}_{\underline{Y}_{3}}+\underline{Y}_{2} \underline{Y}_{3}\right) & \underline{Y}_{4}\left(\underline{Y}_{2} \underline{Y}_{3}+\underline{Y}_{3} \underline{Y}_{4}+\underline{Y}_{1} \underline{Y}_{2}\right)
\end{array}\right|
\end{aligned}
$$

The obtained expressions for the elements of the current distribution matrix are the trees of the graph of the circuit as a function of the natural parameters of the electrical circuit. For all the current distribution coefficients that make up the matrix under study, the denominator is the same, which is determined by the sum of the products of the conductivities of possible trees of the graph.

Possible trees of the graphare shown in the Figure 2.
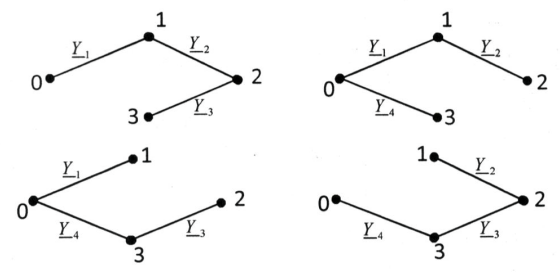

Fig. 2. Closed circuit graph trees.

The numerators of the current distribution coefficients depend on the node of the perturbation application, in the form of a single current, and the geometric image of the circuit.

For example, the numerator of the current distribution coefficient $\underline{C}_{11}$ is formed by the sum of the products of the values of three trees containing the path from the first node to the basic node, through branch 1, shown in Figure 3. 


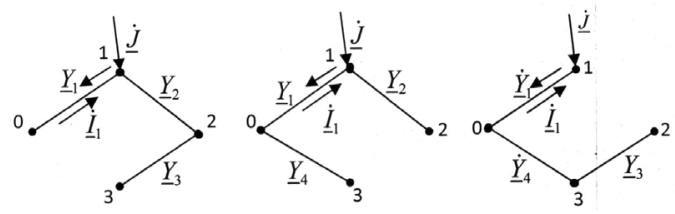

Fig. 3. Graph trees of the numerator $\underline{\mathrm{C}}_{11}$.

The numerators of the coefficients $\underline{\mathrm{C}}_{21}, \underline{\mathrm{C}}_{31}, \underline{\mathrm{C}}_{41}$ are defined likewise.

In the general case, the elements of the current distribution matrix are calculated on the basis of the topological expression

$$
C_{i j}=\frac{\sum_{k=1}^{M} F_{k j}^{1}}{\sum_{k=1}^{N} F_{k}},
$$

where $\sum_{k=1}^{N} F_{k}$-the total value of all possible trees of the graph;

$\sum_{k=1}^{M} F_{k j}^{1}$-algebraic sum of the values of the specific trees of the graph of the i-th branch.

For all current distribution coefficients that are part of the network under study, the denominator is the same, i.e. is determined once by the total value of possible trees of the graph.

\section{Formation of steady-state mode}

The voltage at the nodes relative to the base node is determined by the expression:

$$
\dot{U}=U_{0}+C^{t} Z_{d} C \hat{U}_{d}^{-1} \hat{S},
$$

where $C$-rectangular complex matrix of current distribution coefficients;

$Z_{d}$-diagonal matrix of branches resistances;

$\hat{U}_{d}$ - diagonal matrix of nodal conjugate voltages;

$\hat{S}$-matrix - column of conjugate powers of node loads and generators.

\section{Calculation of the steady state of a real $220 \mathrm{kV}$ network}

The nodalization diagram of the allocated part of the network of the real power system of the Republic of Kazakhstan is shown in Figure 4. The resistance of the branches of the replacement circuit is indicated in the Figure and the total power of the nodes are known. 


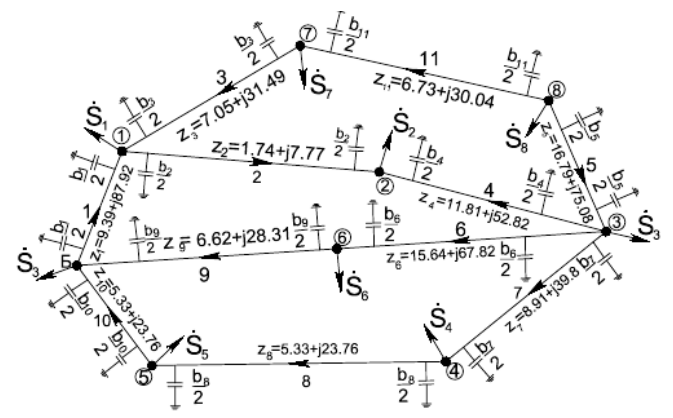

Fig. 4.Electric network nodalization diagram.

\subsection{Formation of a matrix of current distribution coefficients}

All possible trees of the non-directional graph of the original circuit are defined using the theory of structure numbers. To search for and identify specific trees, a special program was developed in the MATLAB environment, on the basis of which the current distribution coefficients were calculated (9).

Calculation results are shown in Tables $1 \mathrm{a}, 1 \mathrm{~b}$.

Table1a.The values of the current distribution coefficients for $C_{i, j=1 \ldots 4}$.

\begin{tabular}{|l|l|l|l|}
\hline$-0.5043+0.0289 \mathrm{i}$ & $-0.4733+0.0272 \mathrm{i}$ & $-0.2631+0.0156 \mathrm{i}$ & $-0.1432+0.0085 \mathrm{i}$ \\
$0.3434+0.0200 \mathrm{i}$ & $-0.5958+0.0188 \mathrm{i}$ & $-0.1823+0.0108 \mathrm{i}$ & $-0.0992+0.0059 \mathrm{i}$ \\
$0.1523+0.0089 \mathrm{i}$ & $0.1224+0.0084 \mathrm{i}$ & $-0.0808+0.0048 \mathrm{i}$ & $-0.0440+0.0026 \mathrm{i}$ \\
$0.3434+0.0200 \mathrm{i}$ & $0.4042+0.0188 \mathrm{i}$ & $-0.1823+0.0108 \mathrm{i}$ & $-0.0992+0.0059 \mathrm{i}$ \\
$0.1523+0.0089 \mathrm{i}$ & $0.1224+0.0084 \mathrm{i}$ & $-0.0808+0.0048 \mathrm{i}$ & $-0.0440+0.0026 \mathrm{i}$ \\
$0.2357+0.0146 \mathrm{i}$ & $0.2504+0.0139 \mathrm{i}$ & $0.3504+0.0087 \mathrm{i}$ & $0.1907+0.0047 \mathrm{i}$ \\
$0.2600+0.0143 \mathrm{i}$ & $0.2762+0.0133 \mathrm{i}$ & $0.3865+0.0069 \mathrm{i}$ & $-0.3339+0.0038 \mathrm{i}$ \\
$0.2600+0.0143 \mathrm{i}$ & $0.2762+0.0133 \mathrm{i}$ & $0.3865+0.0069 \mathrm{i}$ & $0.6661+0.0038 \mathrm{i}$ \\
$0.2357+0.0146 \mathrm{i}$ & $0.2504+0.0139 \mathrm{i}$ & $0.3504+0.0087 \mathrm{i}$ & $0.1907+0.0047 \mathrm{i}$ \\
$0.2600+0.0143 \mathrm{i}$ & $0.2762+0.0133 \mathrm{i}$ & $0.3865+0.0069 \mathrm{i}$ & $0.6661+0.0038 \mathrm{i}$ \\
$0.1523+0.0089 \mathrm{i}$ & $0.1224+0.0084 \mathrm{i}$ & $-0.0808+0.0048 \mathrm{i}$ & $-0.0440+0.0026 \mathrm{i}$ \\
\hline
\end{tabular}

Table1b. The values of the current distribution coefficients for $C_{i, j=5 \ldots 8}$.

\begin{tabular}{|l|l|l|l|}
\hline$-0.0716+0.0043 \mathrm{i}$ & $-0.0775+0.0048 \mathrm{i}$ & $-0.4487+0.0259 \mathrm{i}$ & $-0.3956+0.0229 \mathrm{i}$ \\
$-0.0496+0.0029 \mathrm{i}$ & $-0.0537+0.0033 \mathrm{i}$ & $0.2222+0.0179 \mathrm{i}$ & $0.1066+0.0159 \mathrm{i}$ \\
$-0.0220+0.0013 \mathrm{i}$ & $-0.0238+0.0015 \mathrm{i}$ & $-0.6709+0.0079 \mathrm{i}$ & $-0.5023+0.0070 \mathrm{i}$ \\
$-0.0496+0.0029 \mathrm{i}$ & $-0.0537+0.0033 \mathrm{i}$ & $0.2222+0.0179 \mathrm{i}$ & $0.1066+0.0159 \mathrm{i}$ \\
$-0.0220+0.0013 \mathrm{i}$ & $-0.0238+0.0015 \mathrm{i}$ & $0.3291+0.0079 \mathrm{i}$ & $0.4977+0.0070 \mathrm{i}$ \\
$0.0954+0.0024 \mathrm{i}$ & $-0.1914+0.0030 \mathrm{i}$ & $0.2622+0.0133 \mathrm{i}$ & $0.2874+0.0120 \mathrm{i}$ \\
$-0.1669+0.0019 \mathrm{i}$ & $0.1139+0.0018 \mathrm{i}$ & $0.2892+0.0126 \mathrm{i}$ & $0.3170+0.0109 \mathrm{i}$ \\
$-0.1669+0.0019 \mathrm{i}$ & $0.1139+0.0018 \mathrm{i}$ & $0.2892+0.0126 \mathrm{i}$ & $0.3170+0.0109 \mathrm{i}$ \\
$0.0954+0.0024 \mathrm{i}$ & $0.8086+0.0030 \mathrm{i}$ & $0.2622+0.0133 \mathrm{i}$ & $0.2874+0.0120 \mathrm{i}$ \\
$0.8331+0.0019 \mathrm{i}$ & $0.1139+0.0018 \mathrm{i}$ & $0.2892+0.0126 \mathrm{i}$ & $0.3170+0.0109 \mathrm{i}$ \\
$-0.0220+0.0013 \mathrm{i}$ & $-0.0238+0.0015 \mathrm{i}$ & $0.3291+0.0079 \mathrm{i}$ & $-0.5023+0.0070 \mathrm{i}$ \\
\hline
\end{tabular}

\subsection{Steady-state mode calculations}

Algorithms for the formation of steady-state mode are realized in the MATLAB. All possible graph trees are defined on the basis of the theory of structural numbers, and 
specific trees are based on a specially developed for this purpose algorithm. Calculations of nodal voltage are performed with iterative methods.

A numerical experiment was performed for the design circuit of the allocated part of the network of the real power system of the Republic of Kazakhstan, depicted in Figure 4. The resistance of the branches of the replacement circuit is indicated in the Figure and the total power of the nodes is known. In the process of preparation of the initial data, the steadystate zero-iteration mode is formalized, with subsequent refinement of the node currents to the specified accuracy of the determination of the mode parameters.

Calculation results are shown in Table2.

Table 2.The results of comparative calculations of nodal voltage.

\begin{tabular}{|c|c|c|c|c|c|c|c|}
\hline & \multicolumn{2}{|c|}{$\begin{array}{c}\text { Calculation with } \\
\text { matrix C }\end{array}$} & \multicolumn{2}{c|}{$\begin{array}{c}\text { Calculation according to the } \\
\text { RASTR program }\end{array}$} & \multicolumn{3}{c|}{ Deviations } \\
\hline $\begin{array}{c}\text { Nodes } \\
\text { number }\end{array}$ & $\mathrm{U}, \mathrm{kV}$ & $\delta$, grade & $\mathrm{U}, \mathrm{kV}$ & $\delta$, grade & $\begin{array}{c}\Delta \mathrm{U}, \\
\mathrm{kV}\end{array}$ & $\begin{array}{c}\Delta \mathrm{U}, \\
\%\end{array}$ & $\Delta \delta$, grade \\
\hline 1 & 241.43 & -4.8315 & 240.65 & -4.79 & 0.78 & 0.32 & -0.04 \\
\hline 2 & 241.53 & -4.8433 & 240.78 & -4.81 & 0.75 & 0.31 & -0.03 \\
\hline 3 & 240.89 & -4.3086 & 240.36 & -4.28 & 0.53 & 0.22 & -0.03 \\
\hline 4 & 237.38 & -3.0902 & 237.1 & -3.08 & 0.28 & 0.12 & -0.01 \\
\hline 5 & 235.60 & -1.8466 & 235.45 & -1.84 & 0.15 & 0.06 & -0.01 \\
\hline 6 & 236.27 & -1.9230 & 236.12 & -1.92 & 0.15 & 0.06 & 0.00 \\
\hline 7 & 239.60 & -6.2297 & 238.33 & -6.17 & 1.27 & 0.53 & -0.06 \\
\hline 8 & 239.12 & -6.5359 & 238.03 & -6.49 & 1.09 & 0.46 & -0.05 \\
\hline
\end{tabular}

The allocated section of a real electrical network, in fact, is not very loaded, which is confirmed by the calculations. As can be seen from Table 2, the results obtained by different methods are giving good agreements. It should be noted that the results obtained using the matrix $\mathrm{C}$ are close to real. This is explained by the fact that in the "RASTR" program the reactive powers generated by the line during the calculation are taken into account by constant values and are corrected for the steady-state voltage, whereas in the proposed method, the reactive power of the line is refined at each step of the iteration.

\section{References}

[1] V.A. Stroyev, I.S. Rokotyan, Methods of mathematical optimization in power supply problems (MPEI, Moscow, 1993)

[2] B.Z. Manussov, A.V. Lykin, Yu.M. Sidorkin, News of Higher Educational Institutions of the USSR, Energetics 9, 3 (1974)

[3] O.T. Geraskin, News of Academy of Sciences of the USSR, Energy and Transport 5, 106 (1967)

[4] D. Ahmetbayev, Results in Physics 7, 1644 (2017)

[5] D. Akhmetbayev, A. Akhmetbayev, A. Suleymenov, M. Kolcun, Proceedings of the $9^{\text {th }}$ International Scientific Symposium Elektroenergetika,StaráLesná, Slovak Republic 9,12(2017) 